\title{
Manifestações cutâneo-mucosas da coccidioidomicose: estudo de trinta casos procedentes dos estados do Piauí e Maranhão
}

\author{
Skin and mucous membrane manifestations of coccidioidomycosis: a study of \\ thirty cases in the Brazilian states of Piauí and Maranhão
}

\author{
Antônio de Deus Filho ${ }^{1}$ \\ Alisson de Oliveira Meneses ${ }^{3}$ \\ Aécio Lopes de Araújo Lira ${ }^{5}$
}

\author{
Antônio Castelo Branco de Deus ${ }^{2}$ \\ Andressa Sobral Soares ${ }^{4}$
}

Resumo: Fundamentos: A coccidioidomicose é micose sistêmica usualmente manifesta como infecção benigna de resolução espontânea; porém, uma pequena proporção dos infectados desenvolve quadros progressivos potencialmente fatais, podendo atingir a pele com lesões pleomórficas disseminadas.

OвJетіvos: Identificar e descrever as manifestações cutâneo-mucosas da coccidioidomicose, as ocupações de risco relacionadas à doença e o quadro clínico associado.

MÉTODOs: Estudo realizado entre os anos 2003 e 2006 em 30 pacientes portadores de coccidioidomicose provenientes dos estados do Piauí e Maranhão e diagnosticados por exame microscópico direto, cultivo do escarro ou sorologia de triagem de imunodifusão dupla em gel de ágar, aliados à anamnese e exame físico.

Resultados: Identificaram-se lesões extrapulmonares em 13 casos (43,3\%), com predomínio de manifestações dermatológicas de hipersensibilidade: eritema nodoso (26,6\%), exantema com lesões eritemato-escamosas (26,6\%) e eritema multiforme (23,3\%), além de úlceras de língua (13,3\%) e lábio (6,6\%) e abscesso subcutâneo $(3,3 \%)$. Tais manifestações foram observadas na fase aguda da doença.

CONCLUSÕES: As manifestações cutâneas associadas à infecção respiratória aguda fortalecem a hipótese diagnóstica desta doença, especialmente, em caçadores de tatus ou pessoas expostas à escavação do solo.

Palavras-chave: Coccidioidomicose; Diagnóstico; Epidemiologia; Eritema multiforme; Eritema nodoso; Exantema

Abstract: BACKGRound: Coccidioidomycosis is a systemic mycosis that usually presents as a benign infection. Patients generally recover spontaneously; however, a small proportion of infected individuals develop progressive complications that may affect the skin in the form of disseminated pleomorphic lesions and may become fatal. OBjectives: To identify and describe skin and mucous membrane manifestations of coccidioidomycosis, to identify occupational hazards associated with the disease and to determine its associated clinical presentation.

METHODS: A study conducted between 2003 and 2006 involving 30 patients from the Brazilian states of Piauí and Maranhão with coccidioidomycosis diagnosed by direct microscopy, sputum culture or screening serology using agar gel double immunodiffusion, in association with anamnesis and physical examination.

RESULTS: Extrapulmonary lesions were found in 13 cases (43.3\%), consisting predominantly of dermatological manifestations of hypersensitivity: erythema nodosum (26.6\%), exanthema with erythematosquamous lesions (26.6\%) and erythema multiforme (23.3\%), as well as ulcerations of the tongue (13.3\%), lip ulcers (6.6\%) and subcutaneous abscess (3.3\%). These manifestations were seen during the acute phase of the disease.

ConCLuSIONS: Skin manifestations associated with an acute respiratory infection reinforce the hypothesis of a diagnosis of coccidioidomycosis, particularly in individuals who hunt armadillos or in those exposed to soil excavation.

Keywords: Coccidioidomycosis; Diagnosis; Epidemiology; Erythema multiforme; Erythema nodosum; Exanthema

Recebido em 11.08.2008.

Aprovado pelo Conselho Consultivo e aceito para publicação em 17.12.08

Trabalho realizado na Clínica de Pneumologia do Hospital Getúlio Vargas (CP/HGV), hospital de ensino da Universidade Federal do Piauí (UFPI) Teresina (PI), Brasil.

Conflito de interesse: Nenhum / Conflict of interest: None

Suporte financeiro / Financial funding: : O custeio dos exames complementares realizados neste trabalho foi oriundo do Sistema Único de Saúde (SUS); as demais despesas foram pagas pelos seus autores.

Doutor em Medicina Tropical pela Fiocruz/RJ. Professor associado IV de Pneumologia da Universidade Federal do Piauí (UFPI) ) - Teresina (PI), Brasil. Acadêmico do curso de Medicina da Universidade Federal do Piauí (UFPI) - Teresina (PI), Brasil.

Acadêmico do curso de Medicina da Universidade Federal do Piauí (UFPI) - Teresina (PI), Brasil.

Acadêmica do curso de Medicina Universidade Federal do Piauí (UFPI) - Teresina (PI), Brasil.

Acadêmico do curso de Medicina da Universidade Federal do Piauí (UFPI) - Teresina (PI), Brasil.

CC2010 by Anais Brasileiros de Dermatologia 


\section{INTRODUÇÃO}

As micoses sistêmicas constituem um grupo de infecções adquiridas por meio da inalação de propágulos de fungos dimórficos, causando lesão pulmonar primária com tendência à regressão espontânea no hospedeiro imunocompetente. Conceitua-se como micose pulmonar toda infecção causada por fungo que se expressa com manifestações pulmonares; estão incluídas, além das micoses sistêmicas, as manifestações pulmonares causadas por espécies de Aspergillus (aspergiloses), espécies de fungos da ordem Mucorales (mucormicose), espécies de hifomicetos hialinos e demáceos (hialo e feo-hifomicoses) e espécies de Candida (candidoses ou candidíases). Excetuando-se as manifestações pulmonares por espécies de Candida, todas as demais são adquiridas por inalação, de fonte exógena, abrangendo os fungos dimórficos (agentes das micoses sistêmicas) e os fungos saprófitas oportunistas (agentes das micoses oportunistas). ${ }^{1-6}$

A ocorrência de micoses sistêmicas no semiárido nordestino carece de estudos que possam retratar, de modo consistente, indicadores epidemiológicos de incidência, prevalência, morbidade e mortalidade. $\mathrm{O}$ fato de as micoses pulmonares não serem doenças de notificação compulsória agrava ainda mais o desconhecimento da real magnitude do problema. A busca de melhor sobrevida de hospedeiros imunocomprometidos tornou necessário maior conhecimento acerca das doenças fúngicas, bem como acesso a recursos de diagnóstico micológico seguro. O diagnóstico das infecções pulmonares por fungos é dificultado pelas suas manifestações variadas e frequentemente incaracterísticas. O médico deve considerar a possibilidade de doença fúngica sempre que estiver diante de uma pneumonia subaguda ou crônica que não responda a antibióticos antibacterianos e na qual não tenha sido evidenciada etiologia por bactéria, micobactéria ou vírus. ${ }^{6}$

Manifestações pulmonares causadas por fungos estão presentes com relativa frequência, sobretudo, em grupos etários acima de 30 anos. No Brasil, as micoses que causam manifestações pulmonares crônicas ou que se associam a pneumopatias crônicas de etiologia variada são: coccidioidomicose, paracoccidioidomicose (PCM), aspergilose, histoplasmose e criptococose..$^{2-3,5-6}$

A coccidioidomicose é micose sistêmica que acomete o homem e outros vertebrados. Causada pelo fungo dimórfico geofílico Coccidioides immitis, é adquirida pela inalação de artroconídios infectantes presentes no solo. Recentemente, a partir de estudos de filogenia molecular, demonstrou-se a existência de outra espécie de agente etiológico, que estava "escondida" junto ao $C$. immitis, sendo denominada de
Coccidioides posadasii, em homenagem a Alejandro Posadas, seu descobridor. Assim, conceitua-se, atualmente, que $C$. immitis corresponde aos fungos isolados na Califórnia, sobretudo, do Vale de São Joaquim (EUA), e a espécie $C$. posadasii prevalece em todas as demais áreas endêmicas do continente americano. Dessa maneira, o agente da coccidioidomicose no Brasil denomina-se C. posadasii. ${ }^{7}$

Usualmente, apresenta-se como uma infecção benigna de resolução espontânea, porém, uma pequena proporção dos indivíduos infectados desenvolve quadros progressivos potencialmente fatais. Pode atingir, além dos pulmões, outros órgãos por disseminação hematogênica. ${ }^{8}$

Lesões cutâneas são a localização extrapulmonar mais comum, com predileção pela face e membros, apresentando-se, geralmente, como exantema maculopapular, eritema nodoso, abscessos superficiais, pústulas e lesões granulomatosas. Um mesmo paciente pode apresentar uma ou várias lesões, de aspecto muito variado, com ou sem acometimento de outros órgãos. ${ }^{9}$

A micose é encontrada em regiões áridas e semiáridas do continente americano, sendo o sudoeste dos Estados Unidos da América (EUA) e o norte do México a maior área contígua conhecida com grande prevalência. ${ }^{10}$ Ocorre, também, em áreas endêmicas da América Central e da América do Sul. ${ }^{11}$ Apenas recentemente foi a região semiárida do nordeste do Brasil identificada como área endêmica de coccidioidomicose. ${ }^{12,13}$

O primeiro caso de coccidioidomicose foi identificado em 1891, na Argentina, pelo então estudante de Medicina Alejandro Posadas, em um soldado oriundo do Chaco que apresentava quadro crônico de lesões cutâneas tumorais recorrentes. ${ }^{14}$ Posadas e o patologista Robert Wernicke descreveram um parasita (presente nas lesões) semelhante a protozoários coccídios até então desconhecido. Em 1894, Rixford registrou os dois primeiros casos nos Estados Unidos em imigrantes portugueses recém-chegados à Califórnia que trabalhavam como agricultores no Vale do São Joaquim e, em 1896, Rixford e Gilchrist identificaram nas lesões um parasita similar ao de Posadas. A real natureza desse agente foi desvendada em 1900 por Ophüls e Moffitt, ao descreverem o terceiro caso norte-americano, novamente em imigrante português. Após observarem a regularidade do aparecimento de "mofo" em cultivos, descreveram o agente da doença como sendo um fungo. No Brasil, os primeiros casos foram relatados em 1978 e $1979,{ }^{15,16}$ mas somente em 1998 o País foi incluído no mapa da distribuição geográfica da coccidioidomicose, ${ }^{17}$ após $\mathrm{O}$ relato dos primeiros surtos da forma pulmonar aguda que ocorreram no Piauí e no Ceará. ${ }^{13,18}$ 
A micose já foi diagnosticada em quatro estados brasileiros: Piauí, Ceará, Maranhão e Bahia, sendo que, no Piauí, estado de maior ocorrência, 100 casos já foram identificados. ${ }^{19}$ Além disso, a micose também já foi diagnosticada em cães e tatus (Dasypus novemcinctus), e o C. immitis foi isolado de amostras de solo coletadas de tocas de tatus, nos estados do Piauí 20,21 e do Ceará. ${ }^{22}$

Pelo pouco conhecimento dessa doença em nosso País, é provável que muitos casos sejam erroneamente diagnosticados como pneumonias inespecíficas, tuberculose ou mesmo pneumoconiose/silicose.

\section{MÉTODOS}

O grupo amostral foi constituído por 30 pacientes com quadro clínico de pneumopatias de etiologia a esclarecer, provenientes dos estados do Piauí e do Maranhão e atendidos entre agosto de 2003 e julho de 2006 no ambulatório da Clínica de Pneumologia do Hospital Getúlio Vargas (CP/HGV), hospital de ensino da Universidade Federal do Piauí (UFPI), na cidade de Teresina (PI), Brasil. Realizou-se um estudo observacional, prospectivo, de acordo com as normas que regulamentam a pesquisa em seres humanos contidas nas Resoluções 196/96 e 251/97 do Conselho Nacional de Saúde (Ministério da Saúde do Brasil, 1996).

Os pacientes selecionados para a pesquisa responderam a um questionário pré-elaborado; foram submetidos a anamnese, exame físico, sorologia de imunodifusão dupla (ID), pesquisa direta e cultivo para fungos e bacilos álcool-ácido resistentes (Baar) do escarro e outros espécimes suspeitos de conterem o agente.

\section{Critérios de inclusão}

Pacientes com pneumopatia a esclarecer. Compuseram esse grupo os casos referenciados ou que procuraram espontaneamente a $\mathrm{CP} / \mathrm{HGV}$ e portadores de alterações radiográficas ou tomográficas pulmonares indicativas de processos que necessitaram de investigação diagnóstica com ou sem manifestações respiratórias. Além deles, incluíram-se os casos com manifestações clínicas extrapulmonares, principal- mente, em tegumentos (mucosas, pele), gânglios, sistema nervoso central (SNC), adrenais e outros.

\section{Critérios de exclusão}

Pacientes com infecções respiratórias agudas ou crônicas de etiologia bacteriana ou viral.

\section{RESULTADOS}

Entre os 30 pacientes estudados, 28 (93,3\%) tinham sido expostos recentemente à atividade de risco de caçar e desentocar tatus; um exercia a atividade de plantação/colheita de mandioca e o outro era cavador de poços conhecidos como cacimbões; 29 eram do sexo masculino; 13 eram pardos, dez brancos, cinco negros e dois indígenas. A idade variou de 15 a 82 anos, com média de 37,9 anos; a escolaridade média foi de 2,9 anos; nove pacientes eram analfabetos e um era portador de curso superior.

Observaram-se quatro microepidemias em quatro municípios distintos da região sudoeste do estado do Piauí, acometendo 14 indivíduos. Ocorreram 19 casos isolados, distribuídos em 15 municípios, sendo 17 em pacientes procedentes do estado do Piauí e dois em indígenas da tribo Guajajara do município de Arame (MA) (Figura 1).

No estado do Piauí, 28 pacientes adoeceram: 27 (96,4\%) após exposição à atividade de caçar e desentocar tatus e um por exposição à escavação de poços cacimbão; os outros dois pacientes, procedentes do estado do Maranhão, eram indígenas da tribo Guajajara, do município de Arame: um era caçador de tatu e o outro exercia atividade na plantação e colheita de mandioca. Dentre os pacientes em questão, três (10\%) evoluíram para óbito.

Houve correlação estatisticamente significante entre a atividade de caçar tatus e o aparecimento de coccidioidomicose. A tosse foi o sintoma mais frequente $(100 \%)$, inicialmente seca, tornando-se produtiva com eliminação de esputo mucoide após alguns dias, associada a febre em $26(86,7 \%)$ e a dor torácica em 22 (73,3\%).

Entre os métodos utilizados para o diagnóstico laboratorial (Tabela 1), o mais sensível foi o cultivo do

TABela 1: Exames laboratoriais utilizados para o diagnóstico de coccidioidomicose CP/HGV, Teresina (PI) (8/2003 a 7/2006)

\begin{tabular}{lccc}
\hline Exame & N. de casos & Positivo: N. & $\%$ \\
\hline Cultivo & 30 & 27 & 90 \\
Exame microscópico direto (KOH 10\%) & 30 & 26 & 86,6 \\
Imunodifusão dupla & 28 & 9 & 32,1 \\
Histopatológico & 1 & 1 & 100 \\
\hline
\end{tabular}




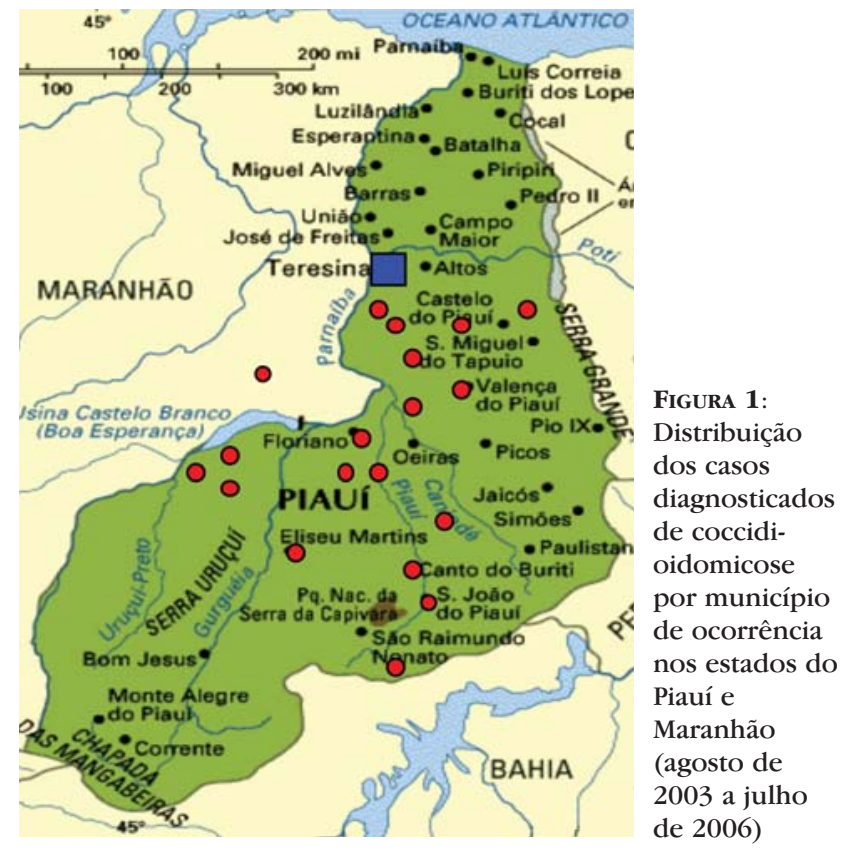

escarro (90\%), secundado pelo exame microscópico direto $(86,6 \%)$.

Manifestações extrapulmonares estiveram presentes em 13 (43,3\%) dos casos, predominando as manifestações dermatológicas de hipersensibilidade, como eritema nodoso $(26,6 \%)$, exantema com lesões eritemato-escamosas $(26,6 \%)$ e eritema multiforme (23,3\%) (Tabela 2 e Figuras 2-5). Alguns pacientes tinham mais de uma alteração cutâneo-mucosa. Com exceção de um caso, todas as manifestações extrapulmonares foram observadas na vigência do diagnóstico, portanto, em evolução aguda da doença.

\section{DISCUSSÃO}

Por sua variedade de achados clínicos, a coccidioidomicose é enquadrada no grupo das "grandes imitadoras", com hanseníase, tuberculose e sífilis. ${ }^{23}$ Tal fato aumenta sobremaneira a importância de se considerarem as manifestações cutâneo-mucosas quando da formulação da hipótese diagnóstica, aliadas à história clínica compatível. Pelo pouco conhecimento da coccidioidomicose em nosso País, muitos casos são, provavelmente, erroneamente diagnosticados como pneumonias inespecíficas, tuberculose ou mesmo pneumoconiose/silicose, especialmente, em pacientes expostos à escavação do solo. ${ }^{19}$

A fácil execução e o baixo custo dos exames micológicos diretos e cultivo reforçam a prioridade a ser dada a esses métodos, respeitados os procedimentos de biossegurança adequados. Outras técnicas de diagnóstico devem ser utilizadas, como a sorologia e, quiçá, técnicas moleculares que poderiam ser disponibilizadas em alguns centros de referência, notadamente, para a região Nordeste do Brasil, onde a doença é endêmica. ${ }^{22}$

O acometimento tegumentar da coccidioidomicose pode ser orgânico ou reativo. As lesões orgânicas contêm os microorganismos cujos espécimes podem ser identificados por exame histopatológico ou por cultura. Essas lesões resultam da disseminação hematogênica para a pele ou, muito mais raramente, de uma infecção cutânea primária. As reações eruptivas, que não contêm organismos viáveis, incluem, mais comumente, EN, exantema agudo generalizado, eritema multiforme e dermatite granulomatosa intersticial. ${ }^{24}$

O eritema nodoso é considerado a reação cutânea mais característica da coccidioidomicose,${ }^{24}$ o que foi confirmado no presente estudo $(26,6 \%$ dos casos, assim como o exantema agudo) (Tabela 2). Todos os pacientes com essa manifestação referiram artralgia concomitante. De uma a três semanas após a instalação da doença, o paciente desenvolve nódulos subcutâneos vermelhos e dolorosos, tipicamente, nas extremidades inferiores (Figura 2). O diagnóstico se baseia, frequentemente, nas características clínicas. A biópsia excisional revela padrão histopatológico de paniculite granulomatosa. Outras causas conhecidas de eritema nodoso incluem sarcoidose, infecção estreptocócica e outras infecções, doença inflamatória intestinal, gravidez e uso de contraceptivos orais. Em pacientes com

TABela 2: Manifestações cutâneo-mucosas da coccidioidomicose pulmonar. CP/HGV, Teresina (PI) (8/2003 a 7/2006)

\begin{tabular}{lll}
\hline Manifestações cutâneo-mucosas $(\mathbf{N}=\mathbf{3 0})$ & $\mathbf{N}^{\mathbf{0}}$ de casos & $\%$ \\
\hline Eritema nodoso & 8 & 26,6 \\
Exantema com lesão eritemato-escamosa & 8 & 26,6 \\
Eritema multiforme & 7 & 23,3 \\
Úlcera de língua & 4 & $\mathbf{1 3}, 3$ \\
Úlcera de lábio & 2 & 6,6 \\
Abscesso subcutâneo & $\mathbf{1}$ & 3,3 \\
\hline
\end{tabular}

Nota: Seis pacientes apresentaram concomitância de eritema nodoso e multiforme e dois mostraram lesão ulcerada de língua associada a eritema nodoso 


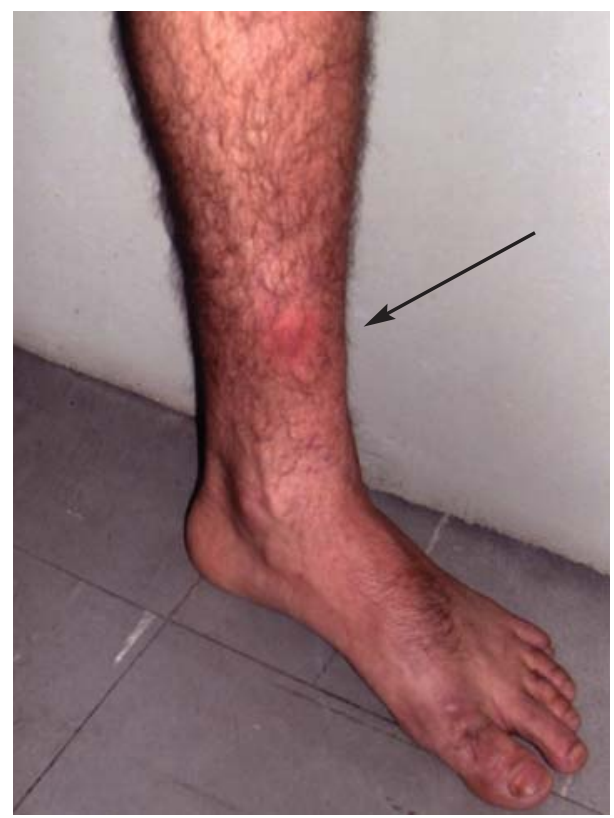

Figura 2:

Eritema nodoso no membro inferior esquerdo (seta)

eritema nodoso, a instalação da erupção coincide com o início da hipersensibilidade tardia. ${ }^{25} \mathrm{O}$ eritema nodoso parece refletir a presença de vigorosa resposta imune mediada por células, o que pode conferir proteção adicional contra o microorganismo. ${ }^{26}$ De fato, todos os pacientes estudados que evoluíram para óbito (10\%) não apresentavam eritema nodoso.

O exantema agudo surge no início do curso da coccidioidomicose - tipicamente, após 48 horas do aparecimento dos primeiros sintomas. ${ }^{27} \mathrm{Em}$ alguns casos, a erupção cutânea foi a queixa principal. Seu surgimento pode preceder o desenvolvimento de anticorpos detectáveis no soro. As lesões cutâneas são clinicamente descritas como máculas, pápulas (Figura 3 ), urticárias ou lesões em alvo, o que resulta em uma aparência clínica que se assemelha à dermatite alérgica de contato ou ao eritema multiforme. O exantema pode persistir por várias semanas e, ocasionalmente, é seguido por descamação palmar e, em alguns casos, por acometimento oral. ${ }^{24}$

Por mais de seis décadas, o eritema multiforme tem sido associado à coccidiodomicose. ${ }^{27}$ Seu quadro clínico parece sobrepor o do exantema agudo. Em ambos os casos, as manifestações ocorrem no início da doença e revelam-se como lesões em alvo, envolvimento oral, prurido e descamação palmar. ${ }^{28} \mathrm{O}$ presente estudo revelou, principalmente, lesões no dorso torácico e em áreas do pescoço e face (Figuras 4 e 5). O exantema agudo pode, clinicamente, mimetizar o eritema multiforme, ainda que as duas entidades difiram histopatologicamente.

Dos 30 pacientes, 28 eram caçadores de tatu, um era cavador de poços e um era lavrador. A associa-

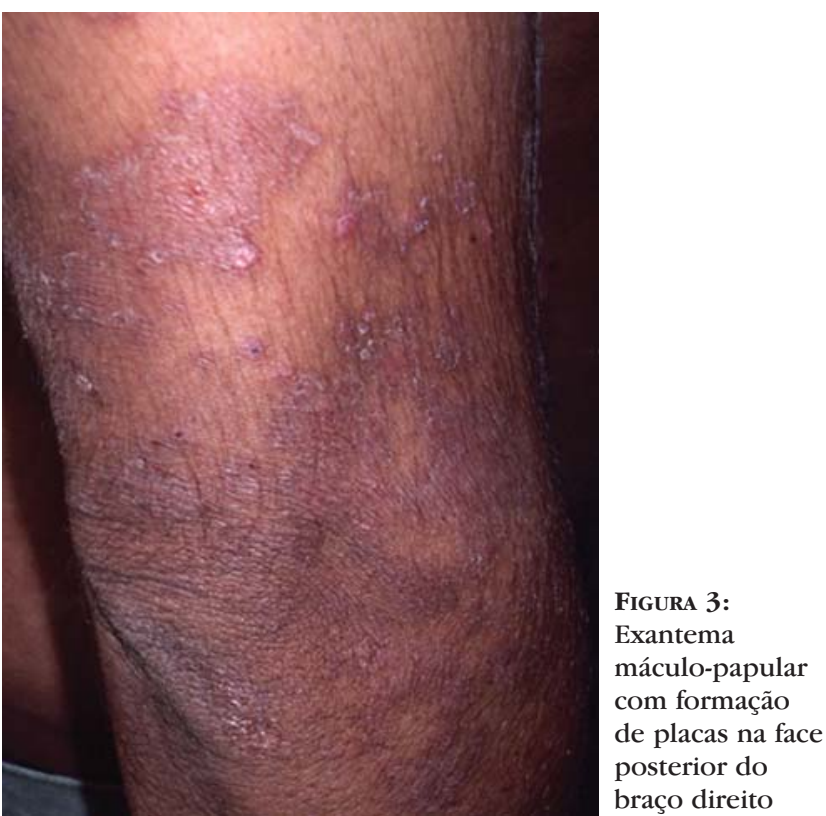

ção entre ocupação de risco e a coccidioidomicose, analisada por meio do teste do qui-quadrado (teste exato), mostrou risco elevado para coccidioidomicose em caçadores de tatu. Portanto, deve-se considerar o diagnóstico de coccidioidomicose diante de quadros infecciosos agudos ou crônicos de apresentação clínica e radiológica variada que surjam após exposição à poeira de terra, seja ela causada por atividade de caça ao tatu ou por remoção de terra devido a outras ocupações.

Estudos descritivos e relatos clínicos têm identificado outros indicadores de risco, como: residência em ambiente rural de área endêmica, exposição a solo, sexo masculino e migração para áreas endêmi-

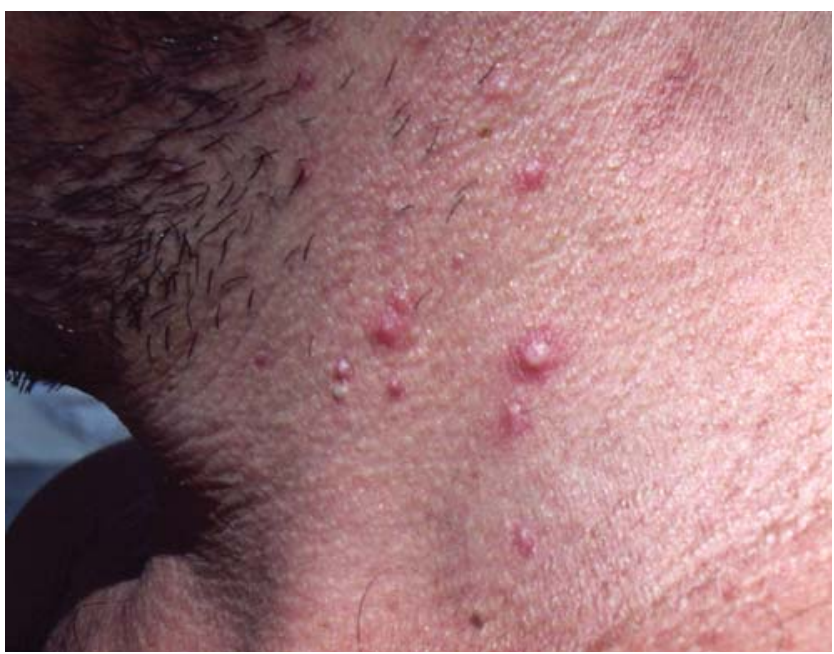

Figura 4: Vesículas e pústulas na região cervical 


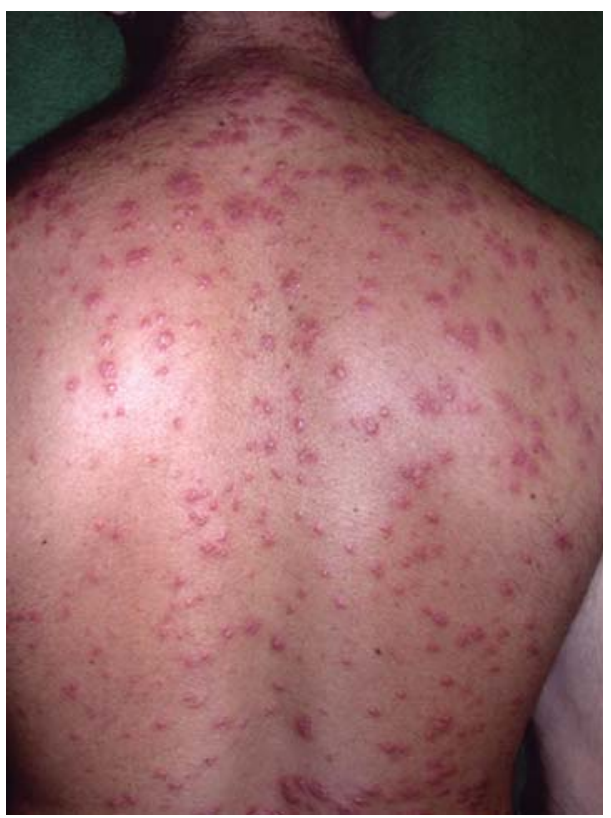

FIGURA 5: Eritema multiforme no dorso

cas. Fatores de risco para doença disseminada incluem as raças negra e asiática.

Os casos em pauta, por representarem formas agudas da doença em que os pacientes foram submetidos a grande inóculo, espelhando manifestação de hipersensibilidade ${ }^{29}$ desencadearam lesões cutâneas em percentagem superior à literatura (26,6\%). Corroborando essa hipótese, todos os quatro pacien- tes da microepidemia ocorrida na cidade de Elesbão Veloso (PI) ${ }^{19}$ tinham manifestações cutâneo-mucosas. Macêdo (2006), ao pesquisar o agente fúngico no solo do mesmo local, encontrou a mais alta taxa de contaminação em amostras obtidas de toca de tatu no estado do Piauí.

O maior conhecimento da enfermidade, incluindo a identificação das ocupações de risco, a transmissão da doença, as características clínicas e as alterações radiológicas, permitiu que médicos pneumologistas e infectologistas, com apoio laboratorial qualificado, confirmassem o diagnóstico em número crescente de casos, destacando-se que, somente no período compreendido entre agosto de 2003 e julho de 2006, dobrou o número de casos conhecidos no estado do Piauí. É muito provável que a micose seja subdiagnosticada e que a sua distribuição geográfica se estenda a outros estados nordestinos ou mesmo a outras regiões brasileiras. ${ }^{13,18,30}$

\section{CONCLUSÃO}

Por se tratar de uma micose sistêmica, a riqueza de achados clínicos pode dificultar o diagnóstico de coccidioidomicose em áreas de baixa ocorrência. $\mathrm{O}$ conhecimento das lesões cutâneo-mucosas e de sua relação com essa doença pode ajudar a diagnosticar corretamente pacientes "suspeitos": expostos à escavação do solo (como caçadores de tatus), com infecção respiratória aguda.

\section{AGRADECIMENTOS}

Ao professor doutor Lauro Lourival Lopes Filho - Dermatologia da Universidade Federal do Piauí (UFPI), pela descrição das lesões cutâneas e mucosas apresentadas pelos nossos pacientes; ao professor doutor Bodo Wanke - Laboratório de Micologia Médica Fiocruz, pelo apoio interinstitucional.

\section{REFERÊNCIAS}

1. Andrade JAF. Avaliação da freqüência de micoses sistêmicas e oportunistas em pacientes com doenças pulmonares: estudo clínico e sorológico no Hospital Otávio Mangabeira [dissertação]. Salvador: Universidade Federal da Bahia; 1987.

2. Chagas PRS. Pesquisa de agentes e anticorpos fúngicos em pacientes com pneumopatia a esclarecer dissertação]. São Paulo: Universidade de São Paulo; 1982.

3. Ferreira-da-Cruz MF. A importância das técnicas de imunoprecipitação na triagem e no diagnóstico da aracoccidioidomicose, histoplasmose e aspergilose. Estudo em população hospitalar do Rio de Janeiro [dissertação]. Rio de Janeiro: IOC-Fiocruz; 1984.
4. Londero AT, Wanke B, Lacaz CS et al. Proposta de iagnóstico e tratamento das micoses profundas com manifestações pulmonares da Comissão de Peritos em Micoses da CNCT e da Comissão Científica da SBPT. Boletim CNCT. 1987;1:53-57.

5. Tarantino AB, Gonçalves AJR, Capone D, Aidé MA, Lazéra MS, Wanke B. Micoses pulmonares. In: Tarantino AB. Doenças pulmonares. 5 ed. Rio de Janeiro: Guanabara Koogan; 2002. p.416-50.

6. Wanke B, Monteiro PCF, Lazéra MS, Capone D, Bethlem EP, Rego AP. Micoses pulmonares. In: Bethlem NM. Pneumologia. 4 ed. Rio de Janeiro: Atheneu; 1996. p.449-77.

7. Fisher MC, Koenig GL, White TJ, Taylor JT. Molecular 
and phenotypic description of Coccidioides posadasii sp. nov., previously recognized as the non-California population of Coccidioides immitis. Mycologia. 2002;94:73-84.

8. Rippon JW. Coccidioidomycosis. In: Medical Mycology. The pathogenic fungi and the pathogenic actinomycetes. 3th ed. W.B. Philadelphia: Saunders Co; 1988. p.443-73.

9. Chiller TM, Galgiani JN, Stevens DA. Coccidioidomycosis. Infect Dis Clin North Am. 2003; 17:41-57.

10. Pappagianis D. Epidemiology of coccidioidomycosis. In: McGinnis MR. Current topics of medical mycology. New York: Springer-Verlag; 1988. p.199-238.

11. Mayorga RP, Espinoza H. Coccidioidomycosis in Mexico and Central America. Mycopathol Mycol Appl. 1970;41:13-23.

12. Wanke B, Lazéra MS, Nucci M. Fungal infections in the immunocompromised host. Mem Inst Oswaldo Cruz. 2000;95(Suppl1):153-8.

13. Sidrim JJC, Silva LCI, Nunes J, Rocha MFG, Paixão GC. Le nord-est brésilien, région d'endémie de occidioidomycose? A propos dune micro-épidémie. J Mycol Méd. 1997;7:37-9.

14. Posadas A. Un nuevo caso de micosis fungoidea con psorospermias. An Circ Med Argent. 1892;15:585-97.

15. Gomes OM, Serrano RRP, Pradel HOV, Moraes NLTB, Varella ALB, Fiorelle AI et al. Coccidioidomicose pulmonar. Primeiro caso nacional. Rev Assoc Med Bras. 1978;24:167-168.

16. Vianna H, Passos HV, Sant'Ana AV. Coccidioidomicose. Relato do primeiro caso ocorrido em nativo do Brasil. Rev Inst Med Trop. 1979;21:51-5.

17. Pappagianis D. Coccidioides immitis. In: Collier L, Balows A, Sussman M. Microbiology and microbial infections. 9th ed. London: Ajello L \& Hay; 1998. v.4. p.357-71.

18. Wanke B, Lazéra MS, Monteiro PC, Lima FC, Leal MJ, Ferreira Filho PL et al. Investigation of an outbreak of endemic coccidioidomycosis in Brazil's northeastern state of Piauí with a review of the occurrence and istribution of Coccidioides immitis in three other Brazilian states. Mycopathologia. 1999;148:57-67.

19. Deus Filho A. Manifestações respiratórias das micoses. Estudo em população com pneumopatia no estado do Piauí [tese]. Rio de Janeiro: Instituto Osvaldo Cruz (Fiocruz); 2007.
20. Eulálio KD. Contribuição ao estudo da coccidioidomicose: identificação de infecção natural de tatus Dasypus novemcintus por Coccidioides immitis [dissertação]. Rio de Janeiro: Instituto Osvaldo Cruz (Fiocruz); 2000.

21. Macêdo RCL. Isolamento e identificação de Coccidioides immitis de amostras do solo relacionadas a surtos de coccidioidomicose [dissertação]. Rio de Janeiro: Instituto Osvaldo Cruz (Fiocruz); 2006.

22. Cordeiro RA. Fenotipagem de cepas de Coccidioides posadasii e desenvolvimento de novas estratégias para o diagnóstico da coccidioidomicose - uma doença emergente no nordeste brasileiro [tese]. Fortaleza: Universidade Federal do Ceará; 2006.

23. Huntington RW Jr. Coccidioidomycosis: a great imitator disease. Arch Pathol Lab Med. 1986;110:182.

24. DiCaudo DJ. Coccdidioidomycosis: a review and update. J Am Acad Dermatol. 2006;55:929-42.

25. Smith CE. Epidemiology of acute coccidioidomycosis with erythema nodosum ("San Joaquin" or "Valley Fever"). Am J Public Health Nations Health. 1940;30:600-11.

26. Braverman IM. Protective effects of erythema nodosum in coccidioidomycosis. Lancet. 1999;353:168.

27. Richardson HB Jr, Anderson JA, McKay BM. Acute pulmonary coccidioidomycosis in children. J Pediatr. 1967; 70:376-82.

28. DiCaudo DJ, Yiannias JA, Laman SD, Warschaw KE. The exanthem of acute pulmonary coccidioidomycosis: clinical and histopathologic features of 3 cases and review of the literature. Arch Dermatol. 2006;142:744-6.

29. Pappagianis D. Coccidioidomycosis. Semin Dermatol. 1993;12:301-9.

30. Wanke B. Coccidioidomicose no Brasil. Infocus América Latina - II Fórum de Infecções Fúngicas na Prática Clínica, São Paulo, Livro de Resumos, 2003. p.55.

\author{
ENDEREÇO PARA CORRESPONDÊNCIA / MAILING ADDRESS: \\ Av. Marechal Castelo Branco, 400 - Apto 1600 \\ Cristo Rei \\ 64014058 Teresina PI \\ Tel.:/Fax: $8632178406 / 99822065$ / 99419233 \\ E-mail: antoniocbdedeus@uol.com.br
}

